\title{
BLENDED LEARNING EN TIEMPOS DE PANDEMIA ¿EXPERIENCIA DE EXITO EN EDUCACIÓN SUPERIOR?
}

\author{
Vanesa Baños Martínez \\ Universidad de Burgos \\ vbanos@ubu.es
}

Recepción Artículo: 27 octubre 2021 Admisión Evaluación: 27 octubre 2021 Informe Evaluador 1: 28 octubre 2021 Informe Evaluador 2: 29 octubre 2021 Aprobación Publicación: 30 octubre 2021

\section{RESUMEN}

La práctica educativa mundial se ha visto sacudida por las restricciones sanitaras derivadas de la pandemia de coronavirus. En estas circunstancias el aprendizaje en línea se ha presentado como una opción interesante y supone el desarrollo de oportunidades y alternativas a explorar en la educación superior (OCDE, 2020). La presente investigación se centra en el alumnado del ámbito universitario y pretende analizar su experiencia de aprendizaje combinado bajo las restricciones derivadas de la pandemia. Este estudio se llevó a cabo mediante un enfoque cuantitativo basado en el método de encuesta sociológica. El instrumento utilizado para la recogida de datos fue un cuestionario anónimo en línea validado con muestra autoseleccionada diseñado ad hoc que se envió a toda la población objeto de estudio, estudiantes del Grado en Pedagogía de la Universidad de Burgos, y se obtuvo una muestra de $n=133$ alumnos. Los resultados muestran que 4 de cada 5 encuestados desean volver a la presencialidad completa y se especifican las diferentes ventajas y desventajas de la enseñanza virtual percibidas por el alumnado. Además, en una escala Likert de 5 grados, se observa una satisfacción moderada con el curso, una atención mejorable en las sesiones virtuales y se constata que el aprendizaje les supone un mayor esfuerzo en formato electrónico. Otros estudios en esta línea también han apuntado una amplia preferencia del alumnado por la presencialidad, subrayan que algunas de las ventajas del aprendizaje virtual son el ahorro de tiempo o la flexibilidad y que entre sus desventajas destacan la falta de interacción o los problemas técnicos. En definitiva, la investigación aporta información relevante que debería tenerse en cuenta para comprender los cambios en curso dentro de la institución universitaria, resolver sus problemas específicos y garantizar, si fuera necesario, su sostenibilidad así como su calidad.

Palabras clave: pandemia; educación superior; eLearning; aprendizaje combinado; satisfacción

\section{ABSTRACT}

Blended learning in times of pandemic, a successful experience in higher education? Global educational practice has been shaken by the health restrictions resulting from the coronavirus pandemic. In these 
circumstances, online learning has emerged as an interesting option and represents the development of opportunities and alternatives to be explored in higher education (OECD, 2020). This research focuses on students at the university level and aims to analyse their blended learning experience under the constraints of the pandemic. This study was conducted using a quantitative approach based on the sociological survey method. The instrument used for data collection was a validated anonymous online questionnaire with self-selected sample designed ad hoc that was sent to the entire study population, students of the Degree in Pedagogy at the University of Burgos, and a sample of $n=133$ students was obtained. The results show that 4 out of 5 respondents wish to return to full face-to-face teaching and the different advantages and disadvantages of e-learning perceived by the students are specified. In addition, on a 5-point Likert scale, moderate satisfaction with the course is observed, with a degree of attention that could be improved in the virtual sessions, and it is noted that learning means more effort for them in electronic format. Other studies along these lines have also pointed to a widespread preference among students for face-to-face learning, underlining that some of the advantages of e-learning are time savings and flexibility, and that some of the disadvantages include the lack of interaction and technical problems. All in all, the research provides relevant information that should be taken into account in order to understand the ongoing changes within the university institution, solve its specific problems and ensure, if necessary, its sustainability as well as its quality.

Keywords: pandemic; higher education; eLearning; blended learning; satisfaction

\section{INTRODUCCIÓN}

El reto más importante al que se han enfrentado los sistemas educativos mundiales en el siglo XXI se presentó de forma sorpresiva a principios de 2020 con la declaración de una nueva y desconocida pandemia causada por un coronavirus. A medida que la crisis sanitaria crecía, causando enormes problemas socioeconómicos a su paso, los sistemas educativos de todo el mundo reaccionaron rápidamente y se adaptaron para garantizar la continuidad de la educación y proteger la seguridad del alumnado y de los agentes educativos apostando principalmente por la enseñanza a distancia (UNESCO, 2021).

El principal aliado para proteger a todos los implicados en el sistema educativo y el marco para ofrecer una alternativa al aprendizaje presencial ha resultado ser la tecnología informática y, en este sentido, Internet se ha convertido en la principal herramienta utilizada para paliar las pérdidas fruto de la no presencialidad.

Así las cosas, durante la pandemia de COVID-19 el aprendizaje en línea se ha presentado como una opción interesante para promover reformas en todo el sistema educativo tradicional que, de lo contrario, hubiesen tardado años en llegar a implantarse. No se puede decir que las universidades hayan realizado un proceso de transformación integral de su docencia hacia el modelo online (García-Peñalvo \& Corell, 2020) pero esta nueva situación supone el desarrollo de oportunidades, retos y alternativas a explorar en la educación superior (OCDE, 2020). Tanto los docentes como el alumnado han tenido que adaptarse y cambiar, por ejemplo, sus comportamientos o su estilo de enseñanza-aprendizaje. Estos cambios han traído consigo múltiples beneficios, si bien también han provocado tensiones, decepciones y frustraciones (Jung et al., 2021) tanto entre el alumnado, muchas veces confundido y perdido entre expectativas y realidad, como entre los docentes, ante el desconocimiento de la efectividad de sus prácticas educativas, por la dificultad en el manejo de las herramientas digitales o por la desigualdad en el acceso a los diferentes recursos electrónicos. En este contexto, para agilizar el impulso de un nuevo escenario educativo, especialmente el que se desarrolla en el ámbito universitario, se requieren intervenciones no solo constructivas sino también creativas, pues estas resolverían problemas concretos y podrían conducir a garantizar la sostenibilidad de este tipo de educación en el tiempo.

El aprendizaje electrónico o eLearning irrumpió a finales de los años 90 a partir del gran desarrollo de las TIC e Internet, pero solo ahora, desde el comienzo de la pandemia de 2020, parece que ha adquirido un protagonismo que le era esquivo, sobre todo a nivel de educación superior, habiendo sabido adaptarse y readaptarse a las nuevas circunstancias.

En el caso de la experiencia analizada en esta investigación, la propuesta para hacer frente a las limitaciones 
de la pandemia está en la línea del blended learning o aprendizaje combinado. Este tipo de aprendizaje permite a los educadores integrar elementos de la instrucción tradicional presencial con modalidades de aprendizaje en línea adaptadas (Garrison \& Kanuka, 2004; Larson \& Sung, 2019; Owston et al., 2012) y se adopta ampliamente en la educación superior con fines variados, utilizando diversas estrategias de implementación (Smith \& Hill, 2019). Como vemos, el aprendizaje combinado tiene potencial para la innovación en los niveles postsecundarios (Garrison \& Vaughan, 2007), en particular dada la frecuente necesidad de equilibrar el espacio del aula y el tamaño de la clase, y el impulso de oportunidades de aprendizaje más flexibles (Berga et al., 2021) aunque su implementación sigue siendo un proceso difícil (Bruggeman et al., 2020). Así las cosas, parece oportuno que el aprendizaje combinado haya sido la modalidad elegida en tiempos de una pandemia que requiere de distanciamiento social y teniendo en consideración, además, que la aceptación del usuario es un prerrequisito para la efectividad de la tecnología (Yardi, 2008) y que la generación más joven está más abierta al uso de la misma (Seman et al., 2019) y suele tener un buen nivel de competencia con las herramientas TIC, especialmente a nivel comunicativo, interactivo y colaborativo (Vázquez-Cano et al., 2020).

\section{OBJETIVOS DE LA INVESTIGACIÓN}

Este estudio tiene como objetivo proporcionar una visión de la experiencia y la satisfacción de los estudiantes hacia la propuesta de aprendizaje híbrido que desde la UBU se desarrolló en el curso académico 2020/2021. Para garantizar la eficacia del proceso de enseñanza-aprendizaje en esta modalidad híbrida y la satisfacción de los estudiantes, los responsables de la toma de decisiones en esta institución superior deberían tener en cuenta los factores estudiados antes de implementar cualquier otra iniciativa de aprendizaje combinado.

En consecuencia, los objetivos de este trabajo son los siguientes

1) Analizar la experiencia de aprendizaje en una modalidad híbrida del alumnado del Grado en Pedagogía poniendo especial atención en sus percepciones y comportamientos.

2) Examinar los predictores de la satisfacción de los estudiantes en el entorno de aprendizaje híbrido implantado en la UBU en el curso 2020/2021 para hacer frente a las restricciones sanitarias.

Hemos planteado como hipótesis principales las siguientes basándonos en la literatura existente:

H1: Las dificultades en el seguimiento de la enseñanza virtual se relacionan negativamente con la satisfacción del alumnado.

H2: La percepción de la necesidad de un mayor esfuerzo en el aprendizaje virtual se relaciona positivamente con el deseo de volver a la enseñanza presencial.

H3: El compromiso con el aprendizaje virtual está vinculado positivamente con la satisfacción del alumnado.

H4: El deseo de volver a la enseñanza presencial tiene una influencia significativa en la satisfacción del alumnado.

\section{MUESTRA Y/O PARTICIPANTES}

La muestra estuvo compuesta por estudiantes de la Facultad de Educación de la Universidad de Burgos, en concreto de los cuatro cursos del Grado en Pedagogía. La población objeto de estudio estaba compuesta por 194 estudiantes y la importancia y el interés de la selección de esta está relacionada con su sensibilización y preocupación por cuestiones de carácter educativo. El margen de error calculado fue de $\pm 5 \%$ y el nivel de confianza del $95 \%$ por lo que la muestra recogida debía ser igual o superior a 130 estudiantes. Se registraron las respuestas de 133 sujetos de todos los cursos, con una representación equilibrada de cada uno de ellos. Más del $91 \%$ del alumnado expuso que residía durante el curso en la propia ciudad de Burgos, frente a poco más del $8 \%$ que 10 hacía en otros lugares. Su edad media, según la información registrada, es de 20,6 años, y su distribución por sexos mostraba una distribución poblacional similar a la de toda la población de estudiantes matriculados en la Facultad de Educación de la Universidad de Burgos (5 \% hombres, 95 \% mujeres). 


\section{METODOLOGÍA Y/O INSTRUMENTOS UTILIZADOS}

La presente investigación se llevó a cabo mediante un enfoque cuantitativo basado en el método de encuesta sociológica. El instrumento utilizado para la recogida de datos fue un cuestionario anónimo en línea que se publicó como formulario de Google, una herramienta gratuita de la citada compañía. Los datos se recogieron entre abril y mayo de 2021. Este método se adoptó debido a las limitaciones de las reuniones presenciales impuestas por la pandemia de COVID-19 y porque, en el caso de la presente investigación, se consideró adecuada la variante de encuesta en línea, ya que la totalidad de la población encuestada tenía acceso a Internet así como un dispositivo electrónico para cumplimentar el cuestionario. El enlace al cuestionario en línea y las directrices para cumplimentarlo se enviaron por correo electrónico en dos ocasiones, con una semana de diferencia, así como mediante mensajería instantánea a través de la aplicación WhatsApp, siendo el enlace distribuido por los delegados o subdelegados de curso. No había obligación de rellenar el cuestionario por parte del alumnado, la actividad era totalmente voluntaria, anónima y confidencial. El tiempo medio necesario para responder al cuestionario fue de 4 minutos, y la tasa de respuesta fue del $69 \%$.

El cuestionario utilizado se validó por un comité de expertos formado por un sociólogo, una psicóloga y una pedagoga experta en eLearning. Por otra parte, el análisis de la consistencia interna del instrumento se ha estudiado mediante el coeficiente de Alfa de Cronbach del conjunto de los 15 ítems del instrumento y el resultado de 0,745 muestra una alta fiabilidad del mismo pues se sitúa a partir 0,70 (Cronbach, 1951; Nunnally \& Bernstein, 1994). El cuestionario constaba de 15 preguntas cerradas, más las preguntas clasificatorias, es decir, las que recogían los datos básicos de los encuestados como la edad, el curso, el género o el lugar de residencia durante el curso. La mayoría de las preguntas cerradas eran de tipo escala, con intervalos de medición de 305 grados. Los datos se analizaron mediante Microsoft $\circledast$ Excel@ para Microsoft 365 MSO.

\section{RESULTADOS ALCANZADOS}

Comenzamos por identificar las dificultades o problemas relacionados con las TIC que el alumnado ha encontrado en el seguimiento de las clases virtuales. En este sentido, comprobamos que más de la mitad de la muestra, un $57,7 \%$, ha presentado problemas con la conexión a Internet y un $46,4 \%$ ha expresado dificultades para poder participar en las sesiones en directo. El curso en el que más problemas se han detectado ha sido primero, un hecho comprensible por el desconocimiento del funcionamiento de las diferentes plataformas al haberse incorporado recientemente a los estudios universitarios. Resaltamos, además, que un $22 \%$ afirma que no se ha encontrado ningún problema relacionado con las TIC y destaca el hecho de que el $80 \%$ del exiguo alumnado masculino expone no haber encontrado ningún tipo de dificultad. Finalmente, es interesante destacar que un 27 $\%$ del alumnado ha detectado dos o más dificultades.

El siguiente aspecto objeto de análisis ha sido identificar las ventajas y las desventajas que presenta el eLearning 0 enseñanza virtual frente a la enseñanza presencial. En relación a las ventajas, un 62,4 \% coincide en señalar que favorece en mayor medida la seguridad sanitaria. Además, un 54,1 \% destaca como ventaja la flexibilidad de espacio y tiempo aun cuando no ha sido el caso en la muestra que se presenta pues estamos hablando de una experiencia de aprendizaje síncrono. Del mismo modo, aunque solo era posible evitar los desplazamientos a la facultad algún día concreto, prácticamente la mitad del alumnado, un 48,2 \%, destaca este aspecto como una ventaja. La comodidad es también otra de las ventajas que más alumnos ha señalado, un 42,9\%, así como la facilidad de acceso a la enseñanza virtual que ha sido seleccionada por el 33,8 \% de la muestra. Concluimos que el $81 \%$ de la muestra entiende que hay más de una ventaja en el eLearning frente al 6,8 \% que considera que este tipo de aprendizaje no tiene ninguna ventaja. 
Figura 1. Ventajas del eLearning o enseñanza virtual frente a la enseñanza presencial Fuente: Elaboración propia.

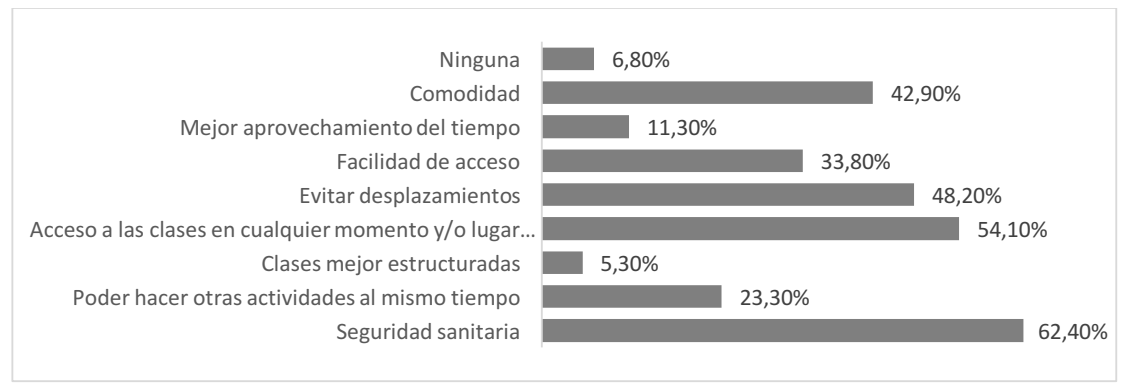

En relación con las desventajas, un 70 \% resalta los problemas técnicos durante la conexión, un 64,1 \% la falta de interacción y un $63,4 \%$ el hecho de que sea más complicado aprender en formato virtual. Con respecto a la falta de interacción, destacar que la plataforma TEAMS que ha sido la utilizada para las sesiones virtuales permite la intervención de los asistentes pero los dispositivos de salida y entrada de audio no favorecen la comunicación con el conjunto del aula por lo que para comunicarse es necesario el docente para que actúe de intermediario. Finalmente, un $44,4 \%$ del alumnado considera una desventaja que falte aplicación práctica y que se ofrezcan en este formato menos explicaciones.

Figura 2. Desventajas del eLearning o enseñanza virtual frente a la enseñanza presencial. Fuente: Elaboración propia.

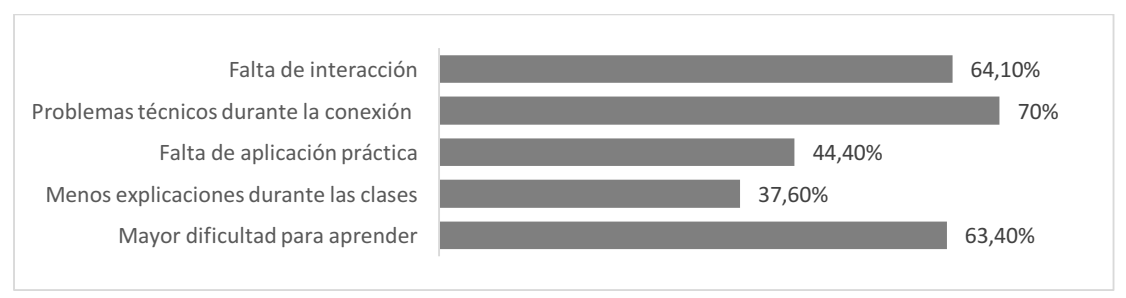

Por otra parte, se ha estudiado la satisfacción del alumnado con respecto a los elementos clave de su formación universitaria. El cálculo de los principales estadísticos descriptivos nos informa de que todos los aspectos presentan una mediana de 3 , en una escala Likert de 5 puntos, siendo la media más baja la relacionada con las clases $(2,93)$ y la más alta la que hace referencia a la adecuación a las circunstancias $(3,17)$. Por otra parte, la desviación estándar o típica de todos y cada uno de los ítems se sitúa en torno a 1, y las distribuciones son más aplanadas que la curva normal. Finalmente, encontramos un coeficiente de asimetría con valor positivo en los ítems de profesorado y clases, que son los peores valorados, mientras que presenta valor negativo en el resto de ítems. La comparación de variables se ha realizado mediante el coeficiente de variación y se ha constatado su baja variabilidad.

Preguntado el alumnado por su forma preferida de aprendizaje, un 68,42 \% afirma que es la modalidad presencial, seguida de la híbrida 0 combinada con un 23,31 \% y únicamente un 8,27 \% afirma que prefiere una modalidad completamente virtual a pesar de estar cursando estudios presenciales. Por otra parte, también se les preguntó cuál era el tipo de docencia que preferían para el curso 2021/2022 y encontramos que la mayoría, un $62,41 \%$, preferiría un curso académico totalmente presencial, seguido de un 29,32 \% que entiende que las cir- 
cunstancias nos obligan a un nuevo curso en modalidad combinada y solo un 8,27 \% desearía que la docencia fuera enteramente virtual. Además, comparando estos datos con los resultados de la pregunta sobre el deseo de volver al aprendizaje totalmente presencial tras el fin de la pandemia, encontramos que el 72,93 \% desea volver a la presencialidad en grado alto, un $14,39 \%$ en grado medio y un $12,78 \%$ en grado bajo. El análisis estadístico de las variables anteriores nos arroja resultados parejos en relación a la media, que se sitúa en torno a 4 en una escala de 5 grados, con un coeficiente de asimetría con valor negativo y un coeficiente de variación alrededor de 29, que interpretamos que es de baja varianza lo que nos muestra que los valores son bastante uniformes. Queda patente que la modalidad presencial es la favorita del alumnado que principalmente echa en falta una mayor interacción y tiene la sensación de que las sesiones virtuales no favorecen su aprendizaje. En este sentido, un 74,44 $\%$ expone que el esfuerzo que tiene que hacer para aprender en las sesiones virtuales es mayor que en las sesiones presenciales. El compromiso del alumnado con las clases virtuales es otro de los aspectos analizado y el mismo se ha examinado a través de una cuestión relacionada con el compromiso durante las clases o nivel de atención y de otra relacionada con la vestimenta. En relación con la primera, únicamente un 21,8 \% afirma que tiene un nivel de atención alto frente a un 31,58 \% que lo describe como igual al del aprendizaje presencial y un $46,62 \%$ que expone que es bajo. En este sentido, queda patente que el alumnado presenta dificultades para mantener el nivel de atención en las sesiones virtuales lo que redunda en el mayor esfuerzo que describen han de hacer para lograr aprender. Por otra parte, cuando se les pregunta hasta qué punto se visten adecuadamente para asistir a las clases en línea y si se toman un tiempo extra para estar presentables como si fueran a asistir a las clases presenciales, una amplia mayoría del 69,9\% responde que nada o poco, un 16,5\% afirma que algo y un $13,6 \%$ expone que bastante 0 mucho. Comprobamos, por tanto, que el compromiso del alumnado es bajo tanto durante las clases virtuales como ante la preparación de las mismas en cuanto a vestimenta se refiere.

\section{DISCUSIÓN}

La pandemia de COVID-19 ha obligado a la adaptación tanto de profesores como de alumnos a las nuevas condiciones que se han traducido, en muchos casos, en una modalidad de aprendizaje eLearning que ha revolucionado el sistema educativo tradicional. La presente investigación ha optado por estudiar la perspectiva de los estudiantes de la Universidad de Burgos con el objetivo de identificar sus actitudes hacia el eLearning, conocer las ventajas y desventajas atribuidas al mismo frente al aprendizaje presencial y su nivel de satisfacción. Los resultados de la investigación desvelan que la muestra analizada tiene una mayor preferencia por el aprendizaje presencial en comparación con el virtual, además el 73 \% de los encuestados declaró mayoritariamente su deseo de volver al formato presencial una vez finalizada la pandemia. Otros estudios en esta línea también han apuntado una amplia preferencia del alumnado por la presencialidad (Adnan \& Anwar, 2020; Gherhes et al., 2021; Radha et al., 2020).

En cuanto a los aspectos positivos del aprendizaje electrónico, la investigación ha demostrado que los estudiantes son particularmente precavidos, considerando la seguridad sanitaria como la principal ventaja, seguida de cerca por la flexibilidad y por la comodidad que supone evitar los desplazamientos. Unos resultados similares se han observado en estudios recientes con estudiantes de la Universidad Politécnica de Timisoara (Gherhes et al., 2021), con estudiantes de Medicina en Polonia (B czek et al., 2021) y con alumnado de España, Italia y Ecuador (Tejedor et al., 2020).

En cuanto a los aspectos negativos, las principales deficiencias del eLearning en comparación con el aprendizaje presencial detectadas por los estudiantes de la muestra fueron los problemas técnicos durante la conexión, la falta de interacción y la mayor dificultad para aprender. Estas desventajas se ven apoyadas por estudios análogos realizados también en el contexto de la pandemia (Adnan \& Anwar, 2020; Miller, 2020; Muthuprasad et al., 2021; OCDE, 2020).

En relación con la satisfacción, el alumnado muestra unos niveles medios, destacando su mayor insatisfacción con las clases y con la organización/coordinación del grado, en esta misma línea de insatisfacción encontramos otras investigaciones (del Arco et al., 2021; Torres Martín et al., 2021). 
En relación con la satisfacción, la confrontación de las cuatro hipótesis formuladas, tras el análisis de la regresión estadística nos informa de que $p>0,05$ y, por tanto, procedemos a rechazar las hipótesis al no poder concluir que las variables presenten algún tipo de relación aun cuando la satisfacción del alumnado sea mayor en quienes están más comprometidos, aquellos que no han tenido problemas durante las clases 0 en los que no han percibido un aumento del esfuerzo.

En el caso de la Universidad de Burgos, la institución ya posee tanto experiencia como una infraestructura básica en el campo de la semipresencialidad por lo que no sería de extrañar que esta iniciativa de aprendizaje combinado, que surgió como consecuencia de la pandemia, pudiera extrapolarse para cubrir diferentes necesidades como la de mayor flexibilidad para compatibilizar la vida laboral y/o personal y la formación universitaria, la adaptación a la diversidad o la personalización de la educación.

\section{CONCLUSIONES}

Los resultados de este estudio dejan claro que el sistema implementado tiene un amplio margen de mejora, pues estudiantes necesitan aprender a autorregularse, adquirir mayor autonomía y responsabilizarse de su propio aprendizaje y los docentes han de adaptarse a una realidad diferente en la que aplicar nuevas estrategias de enseñanza y múltiples herramientas así como redefinir su rol, ahí es donde se ha de poner el énfasis pues la transformación digital de las Universidades va más allá de la mera utilización de tecnología.

Como limitaciones destacaremos que la muestra es relativamente pequeña y el perfil de los encuestados está muy acotado pues proceden de un único grado de la misma institución universitaria. Una futura línea de investigación podría centrarse en si los importantes cambios que se han producido en el plano tecnológico con la implantación de un modelo híbrido de aprendizaje se han acompañado de los necesarios cambios metodológicos. Además, sería de gran interés conocer las experiencias y el punto de vista tanto de los docentes como de los gestores para complementar los resultados de esta investigación.

\section{REFERENCIAS BIBLIOGRÁFICAS}

Adnan, M., \& Anwar, K. (2020). Online Learning amid the COVID-19 Pandemic: Students' Perspectives. Journal of Pedagogical Sociology and Psychology, 2(1), 2020. https://doi.org/10.33902/JPSP

B czek, M., Zaga czyk-B czek, M., Szpringer, M., Jaroszy ski, A., \& Wo akowska-Kapłon, B. (2021). Students' perception of online learning during the COVID-19 pandemic: A survey study of Polish medical students. Medicine, 100(7), e24821. https://doi.org/10.1097/MD.0000000000024821

Berga, K. A., Vadnais, E., Nelson, J., Johnston, S., Buro, K., Hu, R., \& Olaiya, B. (2021). Blended learning versus face-to-face learning in an undergraduate nursing health assessment course: A quasi-experimental study. Nurse Education Today, 96. https://doi.org/10.1016/j.nedt.2020.104622

Bruggeman, B., Tondeur, J., Struyven, K., Pynoo, B., Garone, A., \& Vanslambrouck, S. (2020). Experts speaking: Crucial teacher attributes for implementing blended learning in higher education. https://doi.org/10.1016/j.iheduc.2020.100772

Cronbach, L. J. (1951). Coefficient alpha and the internal structure of tests. Psychometrika, 16(3), 297-334. https://doi.org/10.1007/BF02310555

del Arco, I., Silva, P., \& Flores, 0. (2021). University teaching in times of confinement: The light and shadows of compulsory online learning. Sustainability (Switzerland), 13(1), 1-16. https://doi.org/10.3390/su13010375

García-Peñalvo, F. J., \& Corell, A. (2020). La CoVId-19: ¿enzima de la transformación digital de la docencia 0 reflejo de una crisis metodológica y competencial en la educación superior? En Campus Virtuales (Vol. 9, Número 2). www.revistacampusvirtuales.es

Garrison, D. R., \& Kanuka, H. (2004). Blended learning: Uncovering its transformative potential in higher education. Internet and Higher Education, 7, 95-105. https://doi.org/10.1016/j.iheduc.2004.02.001

Garrison, D. R., \& Vaughan, N. D. (2007). Blended Learning in Higher Education: Framework, Principles, and Guidelines. 
https://books.google.es/books?hl=es\&lr=\&id=2iaR5F0soMcC\&oi=fnd\&pg=PR10\&ots=4Egjq_KLsI\&sig $=\mid v m A y z k Y r L e J Y x S n 45 W A \_v L w N H 0 \& r e d i r \_e s c=y \# v=0 n e p a g e \& q \& f=f a l s e$

Gherhes, V., Stoian, C. E., F rcas, M. A., Stanici, M., \& Marín-Díaz, V. (2021). E-Learning vs. Face-To-Face Learning: Analyzing Students' Preferences and Behaviors. https://doi.org/10.3390/su13084381

Jung, J., Horta, H., \& Postiglione, G. A. (2021). Living in uncertainty: the COVID-19 pandemic and higher education in Hong Kong. Studies in Higher Education, 46(1), 107-120.

https://doi.org/10.1080/03075079.2020.1859685

Larson, D. K., \& Sung, C.-H. (2019). COMPARING STUDENT PERFORMANCE: ONLINE VERSUS BLENDED VERSUS FACE-TO-FACE. En Online Learning (Vol. 13, Número 1). http://www.nosignificantdifference.org

Miller, E. D. (2020). The COVID-19 Pandemic Crisis: The Loss and Trauma Event of Our Time. Journal of Loss and Trauma, 25(6-7), 560-572. https://doi.org/10.1080/15325024.2020.1759217

Muthuprasad, T., Aiswarya, S., Aditya, K. S., \& Jha, G. K. (2021). Students' perception and preference for online education in India during COVID -19 pandemic. Social Sciences \& Humanities Open, 3(1), 100101. https://doi.org/10.1016/j.ssaho.2020.100101

Nunnally, J. C., \& Bernstein, I. H. (1994). Psychometric theory. http://vlib.kmu.ac.ir/kmu/handle/kmu/84743

OCDE. (2020). Covid-19: Global action for a global crisis - OCDE. https://read.oecdilibrary.org/view/?ref=120_120544-8ksud70aj2\&title=Education_responses_to_Covid19_Embracing_digital_learning_and_online_collaboration

Owston, R., York, D., \& Murtha, S. (2012). Student perceptions and achievement in a university blended learning strategic initiative . https://doi.org/10.1016/j.iheduc.2012.12.003

Radha, R., Mahalakshmi, K., Kumar, V. S., \& Saravanakumar, A. R. (2020). E-Learning during Lockdown of Covid19 Pandemic: A Global Perspective. International Journal of Control and Automation, 13(4), 1088-1099.

Seman, S. A. A., Hashim, M. J., Roslin, R. M., \& Ishar, N. I. M. (2019). Millennial learners' acceptance and satisfaction of blended learning environment. Asian Journal of University Education, 15(3), 129-141.

Smith, K., \& Hill, J. (2019). Defining the nature of blended learning through its depiction in current research. Higher Education Research \& Development, 38(2), 383-397. https://doi.org/10.1080/07294360.2018.1517732

Tejedor, S., Cervi, L., Tusa, F., \& Parola, A. (2020). Educación en tiempos de pandemia: reflexiones de alumnos y profesores sobre la enseñanza virtual universitaria en España, Italia y Ecuador. Revista latina de comunicación social, 78, 1-21. https://doi.org/10.4185/RLCS-2020-1466

Torres Martín, C., Acal, C., El Homrani, M., \& Mingorance Estrada, Á. (2021). Impact on the Virtual Learning Environment Due to COVID-19. Sustainability (Basel, Switzerland), 13(2), 582. https://doi.org/10.3390/su13020582

UNESCO. (2021). One year into COVID: prioritizing education recovery to avoid a generational catastrophe UNESCO Biblioteca Digital. https://unesdoc.unesco.org/ark:/48223/pf0000376984

Vázquez-Cano, E., Urrutia, M. L., Parra-González, M. E., \& Meneses, E. L. (2020). Analysis of interpersonal competences in the use of ICT in the Spanish university context. Sustainability (Switzerland), 12(2), 476. https://doi.org/10.3390/su12020476

Yardi, S. (2008). Whispers in the Classroom. Digital Youth, Innovation, and the Unexpected, 143-164. https://doi.org/10.1162/dmal.9780262633598.143 